\title{
Efecto antiinflamatorio y cicatrizante del extracto crudo Gamochaeta americana en heridas post extracción dentaria en Cavia porcellus
}

\section{Anti-inflammatory and healing effect of the crude extract Gamochaeta americana on post dental extraction wounds in Cavia porcellus}

\author{
Clariza Trinidad La Torre Zavaleta ${ }^{1}$, Carla María Ordinola Ramírez ${ }^{2}$, Oscar Joel Oc Carrasco ${ }^{3}$
}

\begin{abstract}
RESUMEN
Para evaluar el efecto antiinflamatorio y cicatrizante del extracto crudo de la lechuguilla (Gamochaeta americana) se realizó un estudio de enfoque cuantitativo, nivel experimental, prospectivo, longitudinal y analítico. En cual se utilizaron 15 cuyes hembras de peso 250-500g de 4 a 6 meses de edad, raza "Perú" (Cavia porcellus), todas ellas con alimentación estándar, los cuales fueron asignadas al azar en tres grupos de 5 cuyes cada uno. Para la recolección de datos se emplearon fichas en la cual se registraron la presencia de inflamación y el proceso de cicatrización por día, la información final fue procesada en Microsoft Excel 2016 y el software de SPSS (Versión 26), para contrastar la Hipótesis se utilizó la estadística ANOVA y el método de Tuckey para comparar los datos recolectados. Los resultados evidencian que al aplicar en concentraciones de $0.25 \mathrm{~g} / 0.5 \mathrm{~mL}$ y $0.5 \mathrm{~g} / 10 \mathrm{~mL}$ del extracto crudo de lechuguilla (Gamochaeta americana) en el lecho quirúrgico, la cicatrización comienza al tercer día post exodoncia y culminan al décimo día, sin signos de inflamación, mientras que si no se aplica ningún extracto el proceso de cicatrización comienzan a partir del quinto día y finaliza a los 15 días post extracción dentaria. Concluyendo que la eficacia del extracto de Gamochaeta americana no solamente se refleja en la cicatrización de un lecho quirúrgico, sino que también se evidencia en la prevención de la inflamación la cual favorece la cicatrización.
\end{abstract}

Palabras clave: Antiinflamatorio, cicatrización, extracto crudo

\begin{abstract}
To evaluate the anti-inflammatory and healing effect of the crude extract of lettuce (Gamochaeta americana), a study with a quantitative approach, experimental level, prospective, longitudinal and analytical was carried out. In which 15 female guinea pigs weighing 250-500g from 4 to 6 months of age, breed "Peru" (Cavia porcellus), were used, all of them with standard feeding, which were randomly assigned in three groups of 5 guinea pigs each. For data collection, files were used in which the presence of inflammation and the healing process were recorded per day, the final information was processed in Microsoft Excel 2016 and the SPSS software (Version 26), to contrast the Hypothesis was used ANOVA statistics and Tuckey's method to compare the collected data. The results show that when applying concentrations of $0.25 \mathrm{~g} / 0.5 \mathrm{~mL}$ and $0.5 \mathrm{~g} / 10 \mathrm{~mL}$ of crude extract of lettuce (Gamochaeta americana) in the surgical bed, healing begins on the third day after extraction and culminates on the tenth day, without signs of inflammation, while if no extract is applied, the healing process begins on the fifth day and ends 15 days after tooth extraction. Concluding that the efficacy of Gamochaeta americana extract is not only reflected in the healing of a surgical bed, but is also evidenced in the prevention of inflammation which favors healing.
\end{abstract}

Keywords: Anti-inflammatory, healing, crude extract

\footnotetext{
${ }^{1}$ Tesista en Estomatología, Universidad Nacional Toribio Rodríguez de Mendoza de Amazonas. Email: catrilaza0130@gmail.com

${ }^{2}$ Asesor: Docente Auxiliar a Tiempo Completo en la Facultad de Ciencias de la Salud, Universidad Nacional Toribio Rodríguez de Mendoza de Amazonas.Email: carla.ordinola@untrm.edu.pe

${ }^{3}$ Co-asesor: Docente Auxiliar a Tiempo Completo en la Facultad de Ciencias de la Salud, Universidad Nacional Toribio Rodríguez de Mendoza deAmazonas.Email: oscar.oc@untrm.edu.pe
} 


\section{INTRODUCCIÓN}

La cirugía bucal es la especialidad más antigua reconocida de la odontología $\mathrm{y}$, por ende, le concierne el diagnóstico y el tratamiento quirúrgico de las enfermedades. La exodoncia es el procedimiento más común que se realiza en la práctica odontológica, después de una intervención quirúrgica en la cavidad oral, surgen diferentes complicaciones que aquejan al paciente como la inflamación post exodoncia y el tiempo de cicatrización.

Intentar reducir al máximo los síntomas secundarios a cualquier intervención quirúrgica bucal, sin interferir el proceso fisiológico de la inflamación que sigue al trauma quirúrgico, debe ser un objetivo primordial para cualquier cirujano. Tras cualquier acto de cirugía bucal, se pone en marcha el proceso inflamatorio que tiene como síntomas fundamentales un cuadro doloroso de la zona intervenida, la tumefacción de partes blandas con la consiguiente deformidad facial, y a veces un cierto grado de trismo. Estos síntomas son muy molestos para el paciente, dependiendo su intensidad de múltiples factores como la complejidad del acto quirúrgico, la duración del mismo, la pericia del cirujano, la existencia o no de yatrogenias, la idiosincrasia del paciente, etc. La minimización de tales manifestaciones incide directamente en la mejora de la calidad de vida de los pacientes, en el grado de satisfacción por el tratamiento, y en la reducción del miedo a este tipo de intervenciones. (Romero, Herrero, Torres, \& Gutiérrez, 2006,pp.205).

La inflamación comienza inmediatamente después de que el tejido es lesionado y en ausencia de factores que la prolonguen, dura aproximadamente de 3 a 5 días. Existen dos fases en la inflamación: vascular y celular. La fase vascular ocurre cuando empieza la inflamación, inicialmente con una vasoconstricción debido a la ruptura celular, con la finalidad de disminuir la pérdida de sangre en el área de la lesión, y a su vez promover la coagulación sanguínea. Pocos minutos después, la histamina y las prostaglandinas E1 y E2, elaboradas por los leucocitos causan vasodilatación y aumento de la permeabilidad al crear pequeñas aberturas entre las células endoteliales, lo cual permite el escape de plasma y leucocitos que migran hacia los espacios intersticiales, facilitando la dilución de los contaminantes y generando una colección de fluidos que es conocido como edema.

(Felzani, 2005)

La cicatrización está producida en tres fases, la primera inflamatoria, siguiendo con la fase proliferativa y, por último, con la fase de remodelado. En odontología se conocen dos tipos de cicatrización, por primera y segunda intención. La primera se logra con el uso de suturas, la segunda se basa en un cierre espontáneo de la herida.

Debido a las complicaciones como alveolitis seca que es la más común y otras es que se busca solucionar de forma rápida la necesidades del paciente, por lo que surge la necesidad de realizar procedimientos que ayuden a acelerar el tratamiento de una forma natural, ya que debido al excesivo uso de fármacos: antibióticos y analgésicos, se está causando gran resistencia bacteriana, así mismo también ocasionando el daño a nivel gastrointestinal afectando así la salud del paciente, por ende se buscan nuevas alternativas que aceleren el proceso de cicatrización en tratamiento quirúrgicos. La medicina tradicional, es utilizada ampliamente y desde tiempos ancestrales en nuestro país. El conocimiento sobre salud, enfermedad, prevención y tratamiento ha sido transmitido de una generación a otra; a través del tiempo. Este saber se basa exclusivamente en la experiencia y en las observaciones.

La Lechuguilla (Gamochaeta americana) es una planta curativa conocida desde hace décadas y algunas investigaciones realizadas refieren que posee una excelente actividad cicatrizante de heridas y desinflamante, así mismo posee una gran actividad contra infecciones, así mismo existen muchas otras plantas que fueron investigadas y que poseen actividades curativas similares a la planta de en estudio. (Pinguil, 2017, pp. 60).

En tal sentido y promovidos por el impulso que hoy en día se da al empleo de recursos naturales en la terapia de diversas enfermedades se procedió a estudiar el efecto cicatrizante y antiinflamatorio de la Lechuguilla (Gamochaeta americana), teniendo como objetivo: Evaluar el efecto antiinflamatorio y cicatrizante del extracto crudo de la Lechuguilla (Gamochaeta americana) como terapia post extracción dentaria en el cuy (Cavia porcellus).

\section{MATERIAL Y MÉTODO}

\section{Modelo experimental y diseño del estudio}

La planta denominada lechuguilla (Gamochaeta americana) fue recolectada por la tarde del día lunes 14 de octubre del 2019 en el distrito de Santo Tomás, provincia de Luya, región Amazonas, para el transporte de la planta se utilizaron bolsas de costalillo para su adecuada ventilación. La elaboración del extracto crudo de lechuguilla (Gamochaeta americana) se llevó a cabo en el laboratorio de Bioquímica de la Facultad Ciencias de la Salud de la Universidad Nacional Toribio Rodríguez de Mendoza bajo la ayuda y supervisión del Mg. Julio Mariano Chávez Milla. 
Se escogieron hojas verdes y aparentemente sanas de la planta Lechuguilla (Gamochaeta americana), dejando de lado aquellas que presentaban insectos, rupturas, manchas y material vegetal extraño. Se pesaron $500 \mathrm{~g}$ de hojas de lechuguilla (Gamochaeta americana), se colocaron en un frasco de vidrio al que se añadió 1 litro de alcohol de $96^{\circ}$, la cantidad suficiente hasta cubrir la muestra por sobre $2 \mathrm{~cm}$ de altura, se mezcló bien, el frasco fue tapado y macerado en un lugar oscuro por un mes, agitándose 15 minutos, 1 vez al día, transcurrido el tiempo de maceración se filtró el macerado usando una bomba de vacío y papel filtro que favoreció la filtración del extracto, luego se procedió a realizar la concentración con ayuda de un Rotavapor $\left(\mathrm{KA}^{\mathrm{R}} \mathrm{RV}\right.$ 05 Basic).

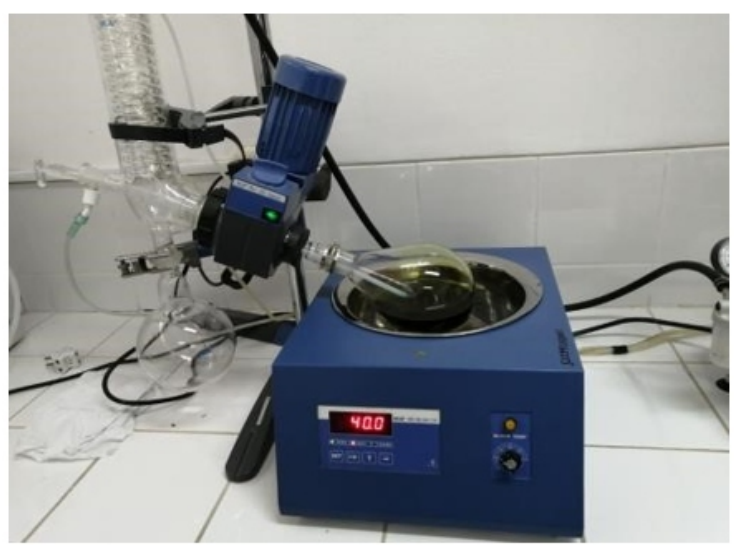

Figura 1. Proceso de concentración del extracto de lechuguilla (Gamochaeta americana).

Aleatoriamente se seleccionaron 15 cuyes hembras sanos, cuyo peso fue de 250-500g de 4 a 6 meses de edad, raza "Perú" (Cavia porcellus), los cuales fueron divididos en 3 grupos:

-Grupo 01: sin aplicación del extracto crudo Lechuguilla (Gamochaeta americana).

-Grupo 02: con aplicación del extracto crudo de Lechuguilla al $0.25 \mathrm{~g} / 0,5 \mathrm{~mL}$ (Gamochaeta americana).

-Grupo 03: con aplicación del extracto crudo de Lechuguilla $0.5 \mathrm{~g} / 1.0 \mathrm{~mL}$ (Gamochaeta americana).

Los cuyes fueron sedados utilizando Xilacina al 2\%, usando solo $0.3 \mathrm{~mL} / \mathrm{kg}$, vía intramuscular, después se esperó 15 minutos para obtener el efecto y se procedió a realizar el acto quirúrgico con ayuda del Co-asesor para tener un mayor campo operatorio y evitar accidentes se procedió al acto quirúrgico.

En la fase quirúrgica se realizó la desinfección externa con Povidona yodada al 10\%, también se precedió a la desinfección intra oral con clorhexidina al 2\%, se anestesió con lidocaína al $2 \%$ infiltrativa sin vasoconstrictor, la Sindesmotomía se hizo con ayuda de un elevador recto mediano, continuando con la luxación de la pieza y posteriormente a la avulsión con un fórceps raigonero o bayoneta.

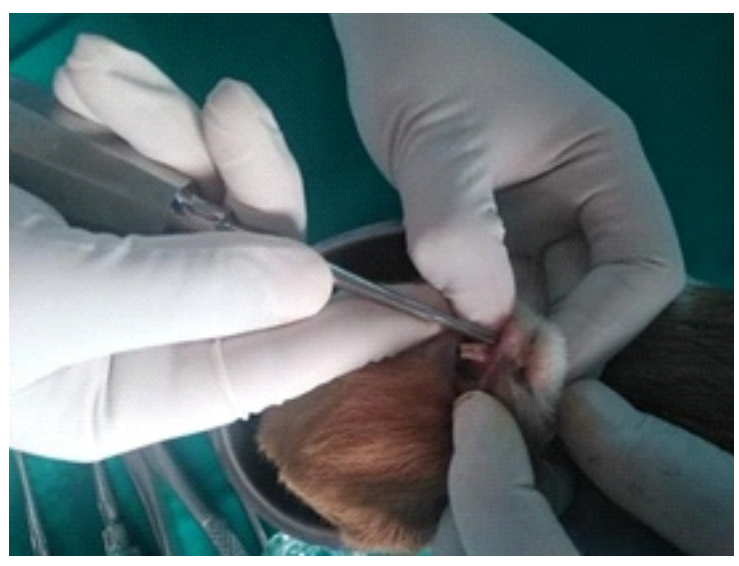

Figura 2. Sindesmotomía con ayuda de un elevador recto mediano.

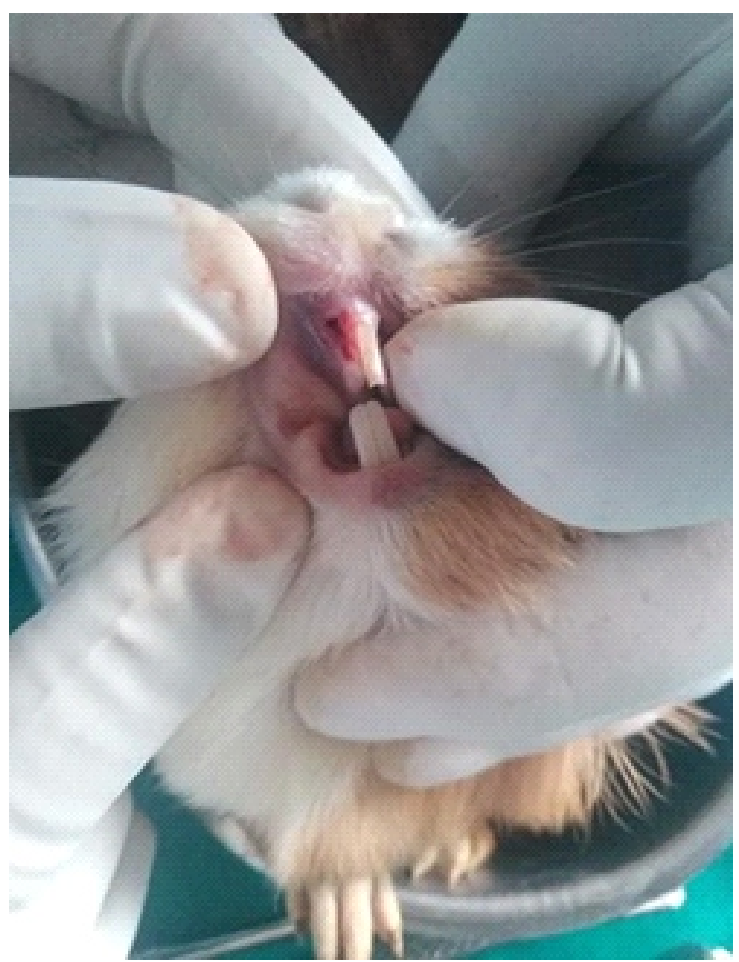

Figura 3. Herida post extracción.

Para la aplicación del extracto crudo se utilizaron hisopos de mando estériles, aplicando hisopadas en el lugar de la extracción del diente, en el grupo 02 se aplicó a una concentración de $0.25 \mathrm{~g} / 0.5 \mathrm{~mL}$ de extracto crudo de manera que cubriera toda la superficie de la herida, lo mismo fue para el grupo 03 al que se aplicó $0.5 \mathrm{~g} / 10 \mathrm{~mL}$ de extracto crudo, la aplicación fue 2 vez al día por 7 días. 


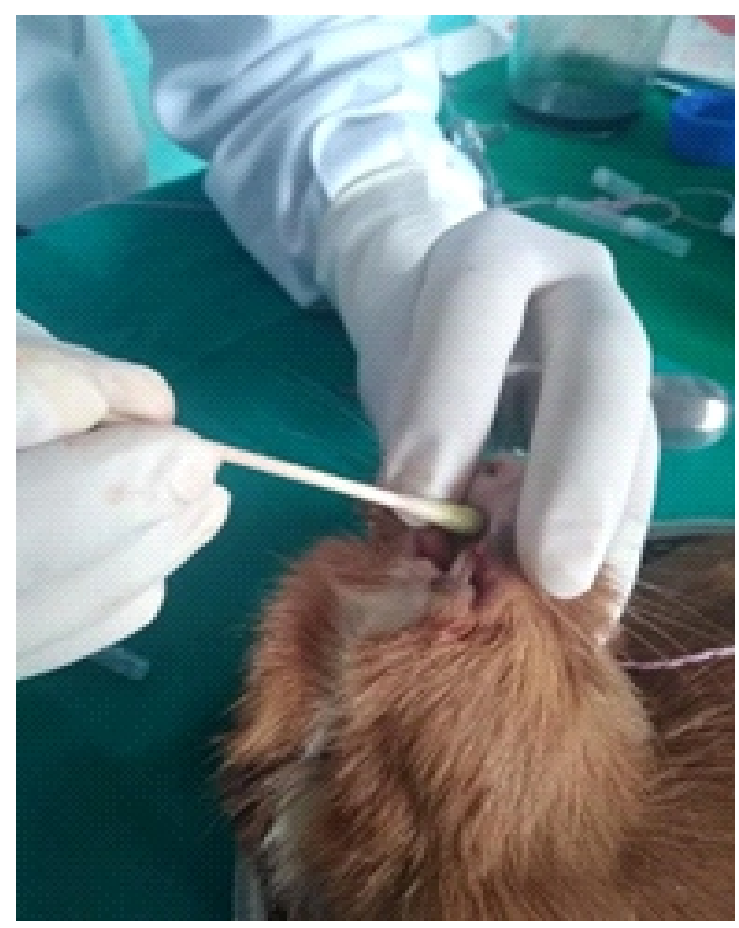

Figura 4. Aplicación del extracto en el lecho quirúrgico.

\section{Análisis estadístico}

Para el ordenamiento, interpretación y Análisis de datos se utilizó Microsoft Excel 2016 y el software de SPSS (Versión 26) y para contrastar la Hipótesis se utilizó la estadística de la prueba de ANOVA y para comparar los datos recolectados mediante el método de Tuckey con un nivel de significancia de $\mathrm{p}$ $>0.05$ (95\% de confiabilidad y 5\% de margen de error). Los resultados se presentaron en tablas y figuras de barras.

\section{RESULTADOS}

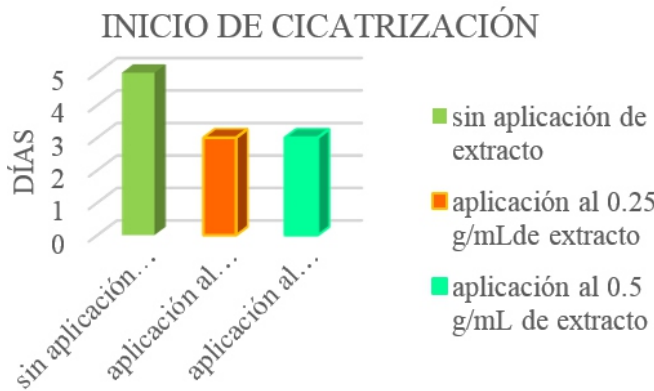

Figura 5. Inicio de cicatrización del lecho quirúrgico en relación a la aplicación del extracto Gamochaeta americana.

Interpretación: En la figura 5 se puede observar los inicios de proceso de cicatrización aplicado al grupo control sin ninguna concentración del extracto de
Gamochaeta americana fue a los 5 días, mientras que al grupo experimental con concentraciones de 0.25 $\mathrm{g} / \mathrm{mL}$ y $0.5 \mathrm{~g} / \mathrm{mL}$ del extracto, el proceso de cicatrización del lecho quirúrgico comenzó a partir de los 3 días.

Aplicado la prueba de Tukey, no se encontró que hay diferencia significativa entre los grupos 02 y 03 de acuerdo a los días de inicio de cicatrización y con respecto al grupo control si se encontraron diferencias significativas ya que la cicatrización inicio a los 5 días post extracción dentaria.

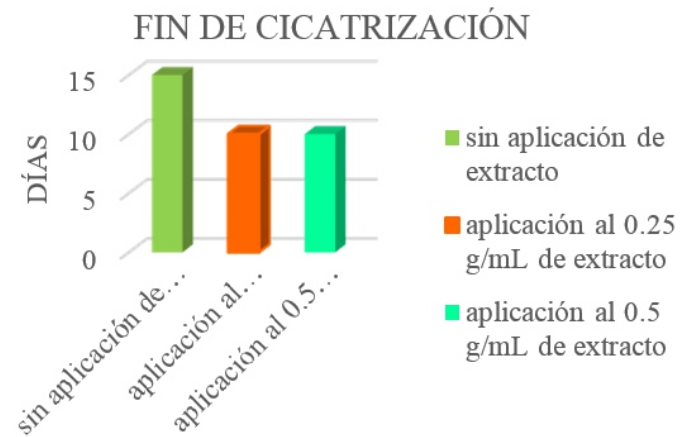

Figura 6. Fin de cicatrización del lecho quirúrgico en relación de la aplicación del extracto de Gamochaeta americana.

Interpretación: En la figura 06 se puede observar que al terminar el proceso de cicatrización del lecho quirúrgico el grupo control al que no se aplicó el extracto (Gamochaeta americana) se dio a los 15 días post extracción, mientras que en los grupos experimentales con aplicación del $0.25 \mathrm{~g} / \mathrm{mL}$ y 0.5 $\mathrm{g} / \mathrm{mL}$ del extracto, se observa que el fin los procesos de cicatrización se da a los diez 10 post extracción dentaria.

\section{DISCUSIÓN}

Al comparar nuestros resultados obtenidos con los estudios realizados por Alcedo (2018) en su trabajo de investigación titulado: "Efecto cicatrizante del extracto hidroalcohólico de las hojas de Gamochaeta purpurea "keto keto", en ungüento aplicados en ratones Mus musculus Balb c", en el cual manifiesta que a mayor concentración de un ungüento a base de Gamochaeta purpurea, la cicatrización es más rápida del lecho quirúrgico, mientras que nuestros resultados muestran que la variabilidad en las concentración de $0.25 \mathrm{~g} / 0.5 \mathrm{~mL} \quad$ y $0.5 \mathrm{~g} / 10 \mathrm{~mL}$ aplicadas de Gamochaeta americana, no muestran diferencias ya que en ambas concentraciones estudiadas se obtuvieron los mismos resultados.

Ríos, M. \& Rodríguez, J. (2013). En su trabajo de investigación titulado: "Evaluación del efecto antiinflamatorio de un gel tópico a base de Gamochaeta americana (queto queto) en animales de experimentación". En el cual se evaluó el efecto 
antiinflamatorio de las partes aéreas de la Gamochaeta americana (Queto Queto) a través de un gel elaborado a base del extracto fluido de Gamochaeta americana (Queto Queto), a las concentraciones de $5 \%, 10 \%$ y $20 \%$ en animales de experimentación, concluyendo que el gel a base de extracto de Gamochaeta americana (Queto Queto) al $20 \%$ obtuvo un porcentaje de inhibición de la inflamación estadísticamente igual al gel a base de extracto de Gamochaeta americana (Queto Queto) al $10 \%$; resultados similares se encontraron en esta investigación demostrando que el efecto antiinflamatorio es similar al aplicar en ambas concentraciones en el los grupos estudiados.

Por otra parte, al comparar nuestros resultados con los obtenidos por Iglesias (2018), en su trabajo de investigación titulado: "Efecto del gel de Matricaria recutita sobre la cicatrización de heridas de mucosa palatina en conejos de la raza nueva Zelanda. En él se demostró que el efecto cicatrizante del gel de Matricaria recutita en herida de mucosa palatina realizada en conejos de la raza Nueva Zelanda”. El gel de manzanilla al 4\% presentó mayor efecto cicatrizante, la cicatrización completa se dio al noveno día mientras que el gel de manzanilla al $2 \%$ se dio a los 19 días, mientras que en nuestro estudio las variación de concentración del extracto de Gamochaeta americana aplicado a los lechos quirúrgicos en Cavia porcellus, tanto al $0.25 \mathrm{~g} / \mathrm{mL}$ como al $0.5 \mathrm{~g} / \mathrm{mL}$ no se encontró diferencias significativas, ya que en ambos casos se evidenció

las manifestaciones de cicatrización al tercer día de la exodoncia dental, además que en ambos casos se obtuvo una cicatrización de la lesión quirúrgica al décimo día.

Mientras que en el estudio realizado por Quispe \& Salazar (2018), en su trabajo de investigación titulado: "Efecto cicatrizante de extracto etanólico de Capsella bursapastoris mediante heridas inducidas en mucosa oral de Cavia porcellus". En el cual obtiene como resultados que el tratamiento con extracto etanólico de Bolsa de Pastor (Capsella bursa pastoris) disminuye el tiempo en el proceso de cicatrización de la mucosa oral a los 7 días. En el cual diferimos ya que con el extracto de Gamochaeta americana se obtiene una cicatrización a los 10 días de tratamiento, y no presentando la lesión quirúrgica inflamación de consideración que retarde o impida el proceso de cicatrización.

\section{CONCLUSIONES}

- El extracto crudo de lechuguilla (Gamochaeta americana) presenta propiedades antiinflamatorias y cicatrizantes aplicadas en heridas post extracción dentaria en el cuy.
- La evaluación de la actividad cicatrizante de acuerdo a los días de aplicación muestra que es menor en los grupos que se aplicó el extracto crudo de lechuguilla (Gamochaeta americana), que en el grupo control.

- El extracto crudo de lechuguilla (Gamochaeta americana) aplicado al $0.25 \mathrm{~g} / 0.5 \mathrm{~mL}$ demostró tener la misma actividad antiinflamatoria y cicatrizante que el que fue aplicado al $0.5 \mathrm{~g} / 1.0$ $\mathrm{mL}$.

\section{REFERENCIAS BIBLIOGRÁFICAS}

Alcedo, C. (2018). "Efecto cicatrizante del extracto hidroalcohólicodelas hojas $d e$ Gamochaeta purpurea (L.) Cabrera "keto keto", en ungüento aplicados en ratones Mus musculus Balbc". Universidad María Auxiliadora. Lima-Perú.

Felzani, R. (2005). Cicatrización de los tejidos con interés en cirugía bucal. A $\quad$ c $\quad$ t a odontológica venezolana ISSN:0001-6365.

Garden., T. M. (2018). Gamochaeta americana(Mill.)Wedd. Obtenido de : D i s p o n i b l e e n : http://www.tropicos.org/Name/2 741289.

Iglesias, S. (2018). Efecto del gel de matricaria recutitasobrelacicatrización $d e$ heridas de mucosa palatina en conejos de la raza nueva zelanda. Universidad Católica los Ángeles chimbote. Trujilli-Perú.

Lazo, J. S. (2018). Evaluación de la actividad anti-inflamatoria $y$ citotóxica in vitro del extracto hidroalcholico de Gnaphalium americanum Mill. Escuela Superio Politècnica de Chimborazo. R i o b a m b a Ecuador.

Morales, T. C. (2018). Efectividad cicatrizante del gel de propoleo y gel del aloe vera sobre heridas post exodoncia simple en rattus rattu. Trujillo-Perú.

Pinguil, S. (2017). Elaboracion de material didactico innovador para la unidad educativa intercultural bilinguequilloac, basado en un catalogo de usos ancestrales de plantas mediciales, para el rescate y revaloración de la cultura cañari. Cuenca- Ecuador.

Pinto, C. \&. (2011). "Efecto cicatrizante de un gel topico a base de cketo cketo(Gamochaeta americana) en animales $d e$ 
experimentación". Universidad Católica de Santa María.Arequipa-Perú.

Quispe, L. (2018). Efecto cicatrizante de extracto etanolico de capsella bursa-pastoris mediante heridas inducidas en mucosa oral de cavia porcellus. Universidad Ncional del Altiplano. Puno- Perú.

Rodriguez, R. \&. (2013). "Evaluación del efecto antiinflamatorio de un gel tópico a base de gamochaeta americana (queto queto) en animales de experimentación". Universidad Católica Santa María. ArequipaPerú.

Romero Ruiz, M., Herrero, M., Torres, D., \& Gutierrez, P. (2006). Protocolo de control del dolor y la inflamación postquirúrgica. Una aproximación racional.RCOE, 205.

Supo, J. (2014). Metodología de la investigacion cientifica para las ciencias de la salud. Arequipa. Perú: Bio estadística EIRL. 